\title{
The Construction of Enterprise Knowledge Market
}

\author{
Mingkui Huo ${ }^{1}$ \\ ${ }^{1}$ Economy and Management School, Changchun University of Science and Technology, Changchun, China \\ Correspondence: Mingkui Huo, Economy and Management School, Changchun University of Science and \\ Technology, Changchun 130025, China. E-mail: huomingkui@163.com
}

Received: November 3, 2014

Accepted: November 16, 2014

Online Published: November 17, 2014

doi:10.5430/ijba.v5n6p73

URL: http://dx.doi.org/10.5430/ijba.v5n6p73

\begin{abstract}
Knowledge is the most important element of enterprise's core competitiveness. The individual and experienced knowledge lies in the minds of workers, this knowledge played a key role in proving efficiency and effectiveness. So, promoting the knowledge sharing is the focus of knowledge management. However, only based on the technology and the leader's command did not have much effect. This article choose a new method to promote the knowledge sharing and and presents countermeasures and suggestion for knowledge management.
\end{abstract}

Keywords: knowledge, knowledge management, knowledge market, construction

\section{Introduction}

In 1998, American scholar Davenport and Prusak puts forward the enterprise internal "knowledge market" theory, emphasize that the enterprise internal "knowledge market" like any other market is a mechanism, that market participants get benefit through the exchange of valuable commodity, the concept of enterprise knowledge market spread quickly in the world. In 2000, Chinese scholar Yingli and Qian Xingsan stress that all kinds of knowledge exchange within the enterprise and transfer fundamentally belong to knowledge transaction, when the knowledge flow freely in the enterprise, its potential value will reflected, the enterprise will have greater cohesion and more abundant knowledge stock.

Subsequently, Dai Jun of the Nanjing University,and Tang Yanhua of the Shanghai Jiao Tong University analysis the pricing mechanism and dynamic mechanism of knowledge market by quantitative model respectively. During that time, more performance for the theoretical analysis and quantitative study are similarly. Based on the research of previous scholars, this paper will be propose some practical guidance strategy of the construction and operation of knowledge market inside enterprises, hoping such research would contribute to enterprise knowledge and knowledge management.

\section{The Traditional Method of Enterprise Knowledge Sharing}

This paper summarized through research on knowledge management and knowledge sharing, and found most traditional research were focused on technology application and sharing strategy shared more knowledge.

\subsection{Technology Application}

There are three kinds technology applied to knowledge sharing mostly: enterprise network, database and group ware technology. Sam Friedman think that although the phone calls and meetings can be used as a e basic channels of knowledge sharing, but the enterprise can not fully reach the knowledge sharing effect, because this method limited communication and conversation, and the intranet and customized search engine will improve knowledge sharing effect, is a good way to obtain the real-time knowledge, combining with the intelligent search engine to promote data mining, knowledge discovery and knowledge flow, can more accurately and timely to promote knowledge sharing.

Knowledge sharing can be combined with ontology method, such as to make network label by ontology method in the special interest groups can help achieving knowledge sharing.You can also achieve knowledge sharing through system integration methods, for example,Sheng Xiaoping designed e-learning fusion model oriented to knowledge management, and integrate the multi-agent system, knowledge management system and electronic learning system together, to achieve the knowledge management and electronic learning by use of information sharing space and University Library during the college.

With the development of information technology, with Blog, RSS, WIKI technology as the representative of the new 
generation network technology has become the enterprise knowledge sharing tool, which can realize the real-time query, but also can through the technology practice of organizational communication atmosphere inside the organization.

\subsection{Organization}

Technology just is a tool, it can provide some basic support however staff will not not share own precious knowledge especially on behalf of their own ability and the authority to everyone just because the convenient. Research shows that, there are lots of barriers in the knowledge sharing process such as cost, psychological land environmental barriers. Break through the barriers must depend on certain organizational culture, trust mechanism, incentive mechanism and the property rights of organization. Therefore, besides technology, also need to he construct organizational knowledge map, establish incentive systems, establish a knowledge sharing type corporate culture and knowledge sharing system and other means to further improve the staff's active participation, promote knowledge sharing, leave a certain organizational strategy, knowledge sharing can only become a laughingstock.

But, whether the application of technology or organizational strategy,during the process of knowledge management, often because of too much emphasis on one aspect while neglecting other factors, into some errors, emerge some strange phenomenon, that is, the same way in small team and to large organizations don't have same work.

So, summarize the Common problems of enterprise knowledge sharing, there are three significant problems, firstly, emphasis on technology but despise the organization culture; secondly, leaders stressed but staff perfunctory; thirdly, a small team have high efficiency but big organization inefficiency.

\section{The Key of Enterprise Knowledge Sharing}

Without a fair return, even if good technology, good example, and strict rules and regulations, employees will not spend time and energy to share their own knowledge to organize. For the initiator, the more difficult he acquit his knowledge, the more he didn't want to share it, because once they are mastered by others, their dominant position will be threatened, these threat include attention degree, salary and promotion chance.

How can we encourage those who know each other or even strangers act with united strength for the common interests. There is a well-known have proved effective every time, the method can make the parties do not know each other exchange all kinds of valuable things, this is the market.

Commodity market have a hundred years of development, the humanity had felt its great capacity for a long time, and massive public knowledge market has already exist, such as the patents, books, articles, the library service organization, online bookstore, database services etc, these have become the source of knowledge and the technical for enterprise. However, the market can help front-line staff exchanges his unique knowledge of the enterprise have not founded.

According to the market economy principle, if we want the market to work effectively, we must ensure that there are an satisfy certain conditions, and have a basic elements such as valuable commodity, right price, exchange mechanism and moderate competition, and as the government regarding the market economy regulation effect, and if we want to ensure knowledge market operation better, also must have the relevant management system and coordination strategy.

\section{Construction of Internal Knowledge Market}

\subsection{Create Valuable Knowledge}

In the process of enterprise knowledge sharing, there are many companies try through conversation or on-line expert system to achieve knowledge transfer, if this way can conduct, is certainly the best, face-to-face communication allows employees who seek knowledge to understand and master the necessary knowledge more quickly. However, the reality in the implementation process is that either demanders cannot successfully find a counterpart expert or experts cannot answer ideally, and then affect knowledge exchange and conversion.

This conversation is more suitable for the knowledge exchange among the minority of an organization, while the charm of knowledge market lies in its ability to greatly expand the dissemination of the original knowledge, thus benefit the entire enterprise. Therefore, there must be a more comfortable way to make buyers and sellers quickly benefit from that knowledge. That means to create some knowledge which can exchange at any time. This knowledge is explicit knowledge created by knowledge owners using their own unique tacit knowledge.

In the market, only those commodity that have value and use value are worth buyers and sellers to invest resources and energy, the internal in the market is the same, if that is ubiquitous common knowledge, it does not require 
transaction, only knowledge that is unique and can bring utility to the buyer will need to trade. Meanwhile, the knowledge acquired through the market must be more profound and more in line with the requirement than the knowledge gained from the other way, also, this knowledge is easier to find and easier to access and absorb.

The key to create the worth exchanging knowledge is not to distinguishing enterprise data, information and knowledge, nor to simply produce a variety of documents within the enterprise to form a database, but to encourage knowledge creators to create. If knowledge demanders can not discuss with experts face to face, enterprises to adopt similar article authorship practices can also increase the creators' power to create high-quality knowledge, only this form of knowledge can be put into market exchange. Such knowledge makes the knowledge demanders understand without communicating with the author face to face.

\subsection{Formulate Price and Protection Mechanisms}

In commodity markets, the compensation obtained by the seller is property, whereas in knowledge market within enterprise, the compensation obtained by the seller is the enhancement of individual reputation within the company. But companies can use a quantitative indicator to evaluate the degrees of reputation and then give material positional and spiritual incentives. The key is to fix a price for knowledge.

Regarding pricing methods of knowledge circulating in the internal knowledge market, whether Davenport or later scholars did not give a specific method can be used to guide practice, because such knowledge is different from such types of knowledge as patents and data knowledge, which can charge a fee through specific price or the number of use. Internal knowledge can not be priced by cost- plus -profit method, and it is not suitable to use the monopoly prices. Also, the most critical question is, during the transfer of knowledge, the purpose of knowledge buyer to gain knowledge is to solve the problem, and to complete the work, the beneficiary is the enterprise, therefore, such compensation of sellers should be paid by the enterprises. The specific payment can be a raise in salary, a promotion or a specific material reward.

Apart from giving the seller a price incentive, enterprise must to protect employees' personal intellectual property rights. The fact that the owner of knowledge and the recipient of various rewards is the author of knowledge rather than anyone else must be clearly identified. Such provision could increase employee's trust in business, therefore, not only to ensure the correct attribution of intellectual property rights, but also to encourage most thoughtful employees to contribute more knowledge of high quality in the future. Only the employees trust the knowledge market, can the market slowly towards prosperity.

\subsection{Strengthen Competition}

As there are many ways inside and outside the enterprise for knowledge demanders to gain required knowledge, in order to operate effectively, the internal knowledge market must be able to provide satisfactory products to make the demand side satisfied. This means to make authors maintain the momentum to continue creating high quality of knowledge. In practice, this momentum will be reflected as a competition unfold by multiple knowledge creators to fight to get recognition.

All markets are prosperous because of competition, knowledge of the market is no exception. Knowledge use quality and popularity to attract readers' attention. Practice shows that if enterprise can give some form of recognition to the author who creates the highest quality or the most popular knowledge, then we can ensure that authors in these enterprises have the momentum to launch aggressive competition in terms of quality and popularity. The best way can be not only judged by experts and executives through sellers' evaluation, but also through the buyer's evaluation and determination, but also determined by statistical downloads which rely on technical methods.

\subsection{Formulate Market Standards}

Buyers and sellers have certain costs incurred in the knowledge market - the time and effort required by creating and acquiring knowledge .For both buyers and sellers, such costs are affordable. If Knowledge market wants to meet this requirement, the relevant standards must be developed to reduce costs and to eliminate concerns.

Standards involve many contents, knowledge used to define the contents of the template is a standard. The requirements used to classify the knowledge in order to facilitating search and retrieval, and what kind of knowledge can enter the market are also standards. Besides, obligations of buyers and sellers must be formulated .For example, the seller want to ensure intelligibility of knowledge, the buyer should evaluate after knowledge is gained; Also standards of specific incentives and the way of rewards need to be made.

\subsection{Market Coordinator}

As the market mechanism needs government to regulate, the internal knowledge markets of enterprises also need 
helps from some coordination staff. It is not only to arrange staff to build and maintain technology platform, but also to arrange someone to implement market standards and norms, and to exercise the function of judgment on the implementation of the provisions. If there is any market coordinator, totally dependent on supplier and demander voluntarily comply with the norms and standards and implement self-management is not feasible, because the supplier and demander are not familiar with these mechanisms, they are short of benefit driven and have no time to do so.

Sometimes these markets coordinators work similar to a commercial database administrator, that is to mark and edit the knowledge of the internal market in order to making querying knowledge more easily, helping readers get enough summary information, or appropriately modifying contents to improve quality on the basis of communicating with the authors. Sometimes markets coordinators can be a network forum administrators, each responsible for a familiar knowledge domain. In each of these domains, there will concentrate a large number of users, their job is to decide in accordance with standards of knowledge which knowledge meets the criteria, which needs to be improved and which can meet the criteria for "good cop", their job also require them to responsible for encouraging relevant experts to develop new creation in their knowledge field of their interest and to convert to text. In addition, the coordinators usually responsible for maintaining and removing outdated content, and checking out whether there is any knowledge gap need to be filled.

\section{Conclusion}

Knowledge management can not depend on the modern information technology, and can not rely on the leader's command, its foundation lies in the establishment and operation of knowledge market, based on the certain technology, relying on the reasonable price mechanism, incentive mechanism, competition mechanism, trust mechanism and the market standard, and excellent market coordination, improve knowledge owners trust on knowledge of the market, to take the initiative to create and share knowledge, also improve the knowledge demand enthusiasm so as to promote knowledge of the prosperity of the market, improve the knowledge sharing effect.

\section{References}

Dai, Jun, \& Zhu, Xiaomei. (2004). Research on the knowledge market inside enterprises. Science of Science and Management of S. \& T., (3), 53-56

Davenport, T. H., \& Prusak, L. (1998). Working Knowledge: How Organizations Manage What They Know. Harvard Business School Press, Boston,MA.

Grant, R. M. (1991). The resource-based theory of competitive advantage: Implications for strategy formulation. California Management Review, (33), 114-135

Jin, Tan, \& David, Yang. (2005). Using Ontology as Scaffolding for Authoring Teaching Materials. International Journal of Distance Education Technologies, 1(3), 81-96.

Sam, Friedman. (1999). Knowledge Sharing Gives Agents an Edge. National Underwriter, 103(19), 9. 